\title{
Mesh Migration Results in Asymptomatic Sigmoid Perforation as Long-Term Complication After Difficult TAPP: a Case Report
}

\author{
Kim R. Liedtke ${ }^{1}$ (D) $\cdot$ Claudia Liedtke $^{1} \cdot$ Annabel Kleinwort ${ }^{1} \cdot$ Paula Döring $^{2} \cdot$ Anne S. Glitsch $^{1} \cdot$ André Schreiber $^{1}$
}

Accepted: 6 April 2020 / Published online: 24 April 2020

(C) The Author(s) 2020

\begin{abstract}
Hernia surgery is the most common surgical procedure worldwide. Complications are very rare and usually manifest in recurrence or chronic pain. We report a rare case of mesh migration 14 years after initially complicated transabdominal preperitoneal plastic for left-sided inguinal hernia. The mesh migration resulted in a covered sigmoid perforation, which was completely asymptomatic and only noticed as a chance finding in a staging CT scan prior to irradiation therapy. However, after the onset of immunosuppressive therapy, an exacerbation of chronic, localized inflammation was expected. Therefore, open surgical anterior rectum resection was performed, and after a short hospital stay, the patient could be discharged home free of complaints. This case report aims to raise awareness of possible long-term complications of hernia repair when using non-absorbable meshes.
\end{abstract}

Keywords Hernia surgery $\cdot$ Mesh migration $\cdot$ Sigmoid perforation $\cdot$ Surgical complications $\cdot$ Transabdominal preperitoneal plastic

\section{Case Report}

Inguinal hernia repair is one of the most common surgical procedures with estimated 20 million procedures per annum worldwide [1]. In addition to open hernia repair including reinforcement via mesh (e.g., Lichtenstein technique), transabdominal preperitoneal plastic (TAPP) is a widely accepted method for minimally invasive hernia repair. The International Endohernia Society (IEHS) values the latter as effective surgical treatment for inguinal hernia [2]. Open procedure or minimally invasive approach, however, hernia surgery is both easy and safe with a 30-day-mortality comparable with the population-standardized mortality rate and rare wound infections. The most common long-term complications are hernia recurrence, chronic pain, and infertility [3]. All these events are scarce, but very rarely, other complications can arise.

This article is part of the Topical Collection on Surgery

Kim R. Liedtke

kim.liedtke@med.uni-greifswald.de

1 Department of General, Visceral, Thoracic and Vascular Surgery, University of Greifswald, Sauerbruchstraße, 17475 Greifswald, Germany

2 Institute of Pathology, University of Greifswald, Friedrich-Loeffler-Straße 23e, 17475 Greifswald, Germany
A 57-year-old patient presented with CT-suspected perforation in the sigmoid colon. However, the patient was in a good condition, and vegetative symptoms were negated. There were no known allergies, and drug ingestion was limited to antihypertensive drugs (i.e., bisoprolol, ramipril, torasemide, pantoprazole, and alendronate).

Fourteen years ago, he underwent laparoscopic hernia repair via TAPP, after rapidly progredient inguinal hernia left sided. Intraoperatively, the sigmoid was displaced in the hernial sac (i.e., sliding hernia) and most likely fused with the peritoneum in a long-stretched post-inflammatory manner, so that a wedge resection (ENDO-GIA $45 \mathrm{~mm}$, Medtronic, Ireland) was necessary for mobilization. Next, the hernia gate was obturated via mesh (Prolene $10 \times 15 \mathrm{~cm}$, TiMESH, GfE Medizintechnik, Germany), which was fixed by fibrin glue (Tissucol, Baxter, USA). Finally, peritoneum was closed with absorbable ligating clips (Lapro-Clip, Covidien, Ireland). Anastasis was prolonged by constipation and stomach pain with sick leave for 3 months and weight loss of $10 \mathrm{~kg}$. However, the condition returned, and he regained body weight without further diagnostic or treatment. One year further, he underwent complication-free TAPP (Ultrapro mesh) on the opposite side. According to the OP report (operated externally), the left side was unremarkable at this time. At the age of 55 years, following an acute bradycardic episode, dilated cardiomyopathy was diagnosed and consequently a cardio defibrillator implanted. However, recent endosonographic 


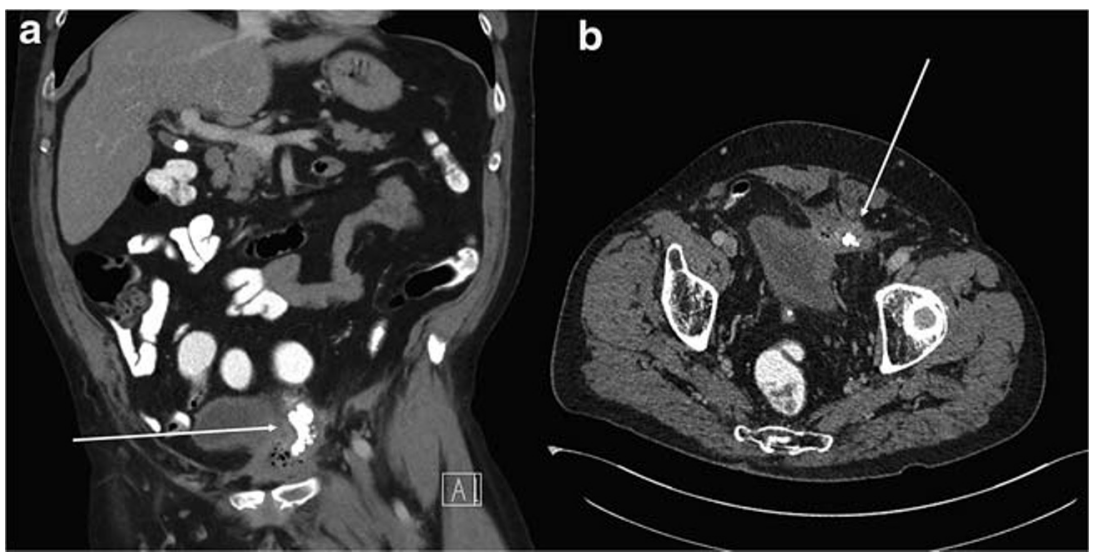

Fig. 1 CT scan with suspected finding at the sigmoid colon. The finding was interpreted as chronic suture insufficiency with covered perforation in the area of the sigmoid colon. The hyperdense area was most likely to be considered coprolith. In close relationship to the bladder occurs a

controls demonstrated normal ejection fraction. One year later, urologic check-up showed elevated PSA levels. Staging revealed prostate cancer (Gleason score, $4+5=9$ ) with osseous metastases (cT2b cN0 cM1b). Due to the metastases and the cardiac prognosis, urologic decision was made to perform irradiation combined with antiandrogenic therapy. Furthermore, CT showed an unclear process in the sigmoid colon (Fig. 1), most likely a covered perforation and embedded coprolith in the sigmoid colon. Before initiating irradiation, anyhow, the sigmoid finding had to be clarified.

Therefore, we performed a colonoscopy that was extremely difficult due to severe angulations of the sigmoid colon. At $30 \mathrm{~cm}$ ab ano, the fibrin-occupied, locally limited insufficiency cavity was found; the supposed coprolith turned out to be the migrated mesh after TAPP 14 years ago (Fig. 2). An endoscopic removal was not successful. Therefore, an open surgical anterior rectum resection with partial parietal peritonectomy was performed. A large paraluminal and prevesical abscess cavity was removed in which the shrunken mesh was found partly migrated into the sigmoid colon and with close contact to the urinary bladder. Histology revealed a moderate florid and chronic fistulizing inflammation in the colon specimen (Fig. 3). chronic inflammatory wall widening. Images in coronary (A) and axial alignment (B). Arrow indicates the migrated mesh and insufficiency cavity containing air. CT scan is enhanced by oral, rectal, and intravenous applicated contrast agent (iodine based)

In the microbiological examination, positive results were found for Proteus vulgaris et penneri, Enterococcus species, Escherichia coli, and greening Streptococci. However, due to missing signs of generalized infection, the patient did not receive antibiotic therapy. The postoperative course was inconspicuous, and 5 days after surgery, rectoscopy revealed timely healing of the anastomosis, followed by the discharge of the patient.

Due to the high number of hernia repairs, there is great evidence for the common operation techniques. Concentrated techniques using a mesh are superior to those that can be summarized as "non-mesh" approaches (e.g., Shouldice technique) [4]. Nonetheless, mesh-related complications like infection or chronic pain are possible drawbacks after hernia surgery. Another complication represents the mesh migration; as a result of which, the mesh can erode surrounding tissue. Fortunately, such an event is rarely observed. Most case reports are related to erosion into the urinary bladder, resulting in dysuria, recurrent urinary tract infections, or suspected cancer [5-9]. Some cases have also been described in which the mesh has migrated into the intestine, depending on the location of the hernia to the cecum [10-12] or sigmoid colon $[13,14]$, respectively. In one case,
Fig. 2 Colonoscopy revealed the mesh migration as the cause of the perforation. A Endoscopic view on a large insufficiency cavity in the sigmoid colon. B After extensive rinsing, some food remains $(*)$ and the adhered TAPP mesh (arrow) are visible in this area

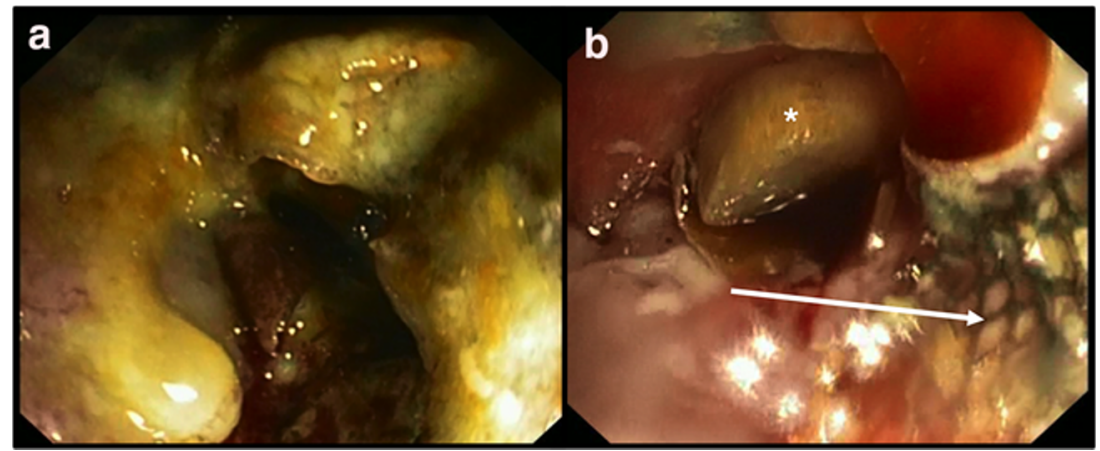




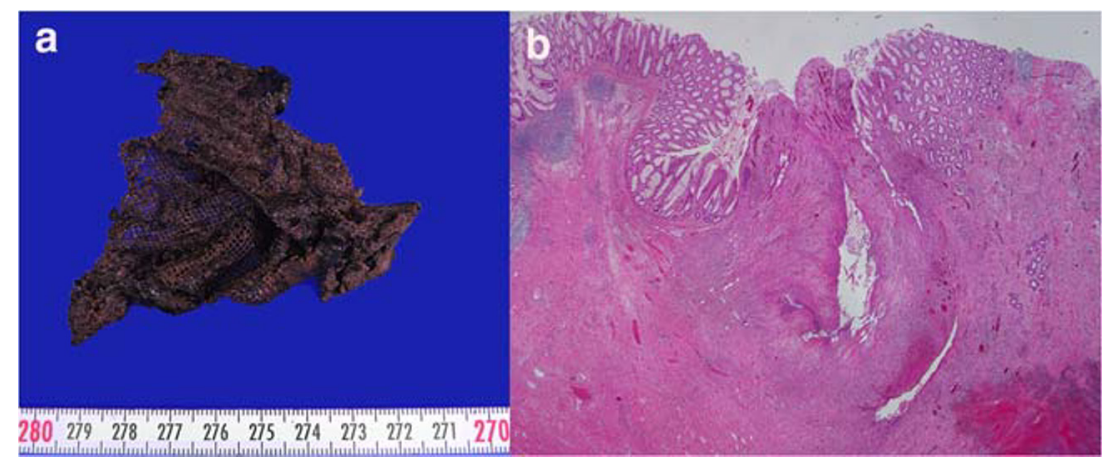

Fig. 3 The migrated mesh and histological colon specimen. A Shown is the approximately $7 \mathrm{~cm}$ large, to a lump adhered mesh, covered with old blood clots. B The mucosal continuity is interrupted, and a deep gap runs through the intestinal wall. At higher magnification (not shown), the accompanying inflammation is visible. The lower edge of the picture corresponds to a length of $9.95 \mathrm{~mm}$ mesh migration even resulted in mechanical bowel obstruction [15]. Because of the rarity, however, these cases include open inguinal hernia repair and ventral hernias. Additionally, all patients became present due to the symptoms associated with the migration.

Here, the mesh migration became conspicuous coincidentally 14 years after hernia repair. This is a relatively long latency, since most complications from mesh migration have already become noticeable in the period between 6 months and 3 years after initial hernia repair [9]. As another special feature of this case compared with those described in the literature, however, this patient did not show any symptoms, just like hernia recurrence, increased urinary infections, abnormal stool, abdominal pain, or inguinal discomfort. To the knowledge of the authors, this is the first report of an asymptomatic mesh migration. Anyhow, we expect the migrated mesh and the resulting abscess formation to become clinically present, at least with start of the irradiation.

Even 30 years after implementation of mesh containing approaches in laparoscopic hernia surgery, the reasons for implant migration are still unclear. What might have contributed to mesh migration here? A crucial role is attached to the mesh fixation. Here, fixation was done by fibrin glue, which is meant to be safe in endoscopic hernia repair [16]. Actual guideline recommends fixation of mesh after TAPP in primary inguinal hernia even only for large hernias (i.e., L III or M III following IEHS classification) [17].

Postoperative convalescence was delayed (obstipation, weight loss), which may have been a symptom of early mesh migration. Furthermore, the patient had a physically demanding job (construction) and has quickly returned to physical strain following the surgery. However, 1 year later during the laparoscopic hernia repair on the opposite side, a normal finding had been made, and a (traumatic) tearing of the mesh would have been more likely in the first weeks after surgery. With regard to the medication of the patient, alendronate is known to cause gastrointestinal side effects up to ulceration. However, it is very unlikely to be causative, since complications are frequently in upper gastrointestinal system [18]. In this case, wedge resection, which was performed at initial hernia repair due to adhesion, may play a decisive role in the genesis: the stapling suture could lead to erosion in the area of the abdominal wall and consecutive mesh detachment. However, literature recherche revealed no comparable report on this specific topic.

Regardless of the cause of mesh migration, one possible answer could be the implantation of (partially) absorbable meshes [19]. However, despite equivalent results, these still represent an exception due to the higher costs [20]. Further studies, especially on long-term outcomes as well as lower product prices, seem to be the prerequisite for change in practice. After all, hernia surgery is globally established, and both the open and the laparoscopic procedures and the meshes used are safe and effective with simultaneously low complication rate. However, mesh migration can be clinically "silent" for a long duration, as demonstrated, suggesting a higher dark figure. Therefore, physicians should be aware of the possibility of mesh migration and consider implantation of degradable mesh in case of difficult TAPP.

Funding Information Open Access funding provided by Projekt DEAL.

\section{Compliance with Ethical Standards}

Conflict of Interest The authors declare that they have no conflict of interest.

Ethical Approval Approval from an institutional board review is not required for a case report.

This article does not contain any studies with human participants or animals performed by any of the authors.

Informed Consent Informed consent was obtained from the patient for publication of this case report and corresponding images.

Open Access This article is licensed under a Creative Commons Attribution 4.0 International License, which permits use, sharing, adaptation, distribution and reproduction in any medium or format, as long as you give appropriate credit to the original author(s) and the source, provide a link to the Creative Commons licence, and indicate if changes were 
made. The images or other third party material in this article are included in the article's Creative Commons licence, unless indicated otherwise in a credit line to the material. If material is not included in the article's Creative Commons licence and your intended use is not permitted by statutory regulation or exceeds the permitted use, you will need to obtain permission directly from the copyright holder. To view a copy of this licence, visit http://creativecommons.org/licenses/by/4.0/.

\section{References}

1. Bay-Nielsen M, Kehlet H, Strand L, Malmstrøm J, Andersen FH, Wara P, et al. Quality assessment of 26304 herniorrhaphies in Denmark: a prospective nationwide study. Lancet. 2001;358(9288):1124-8.

2. Bittner R, Montgomery MA, Arregui E, Bansal V, Bingener J, Bisgaard T, et al. Update of guidelines on laparoscopic (TAPP) and endoscopic (TEP) treatment of inguinal hernia (International Endohernia Society). Surg Endosc. 2015;29(2):289-321.

3. Jenkins JT, O'Dwyer PJ. Inguinal hernias. BMJ. 2008;336(7638): 269-72.

4. Kulacoglu H. Current options in inguinal hernia repair in adult patients. Hippokratia. 2011;15(3):223-31.

5. Hume RH, Bour J. Mesh migration following laparoscopic inguinal hernia repair. J Laparoendosc Surg. 1996;6(5):333-5.

6. Agrawal A, Avill R. Mesh migration following repair of inguinal hernia: a case report and review of literature. Hernia. 2006;10(1):79-82.

7. Novaretti JP, et al. Migration mesh mimicking bladder malignancy after open inguinal hernia repair. Hernia. 2012;16(4):467-70.

8. Bodenbach M, Bschleipfer T, Stoschek M, Beckert R, Sparwasser C. Intravesical migration of a polypropylene mesh implant 3 years after laparoscopic transperitoneal hernioplasty. Urologe A. 2002;41(4):366-8.

9. Hamouda A, Kennedy J, Grant N, Nigam A, Karanjia N. Mesh erosion into the urinary bladder following laparoscopic inguinal hernia repair; is this the tip of the iceberg? Hernia. 2010;14(3):317-9.

10. Goswami R, Babor M, Ojo A. Mesh erosion into caecum following laparoscopic repair of inguinal hernia (TAPP): a case report and literature review. J Laparoendosc Adv Surg Tech A. 2007;17(5): 669-72.

11. Aziz F, Zaeem M. Chronic abdominal pain secondary to mesh erosion into ceacum following incisional hernia repair: a case report and literature review. J Clin Med Res. 2014;6(2):153-5.

12. Ojo P, Abenthroth A, Fiedler P, Yavorek G. Migrating mesh mimicking colonic malignancy. Am Surg. 2006;72(12):1210-1.

13. Chan RH, Lee KT, Wu CH, Lin WT, Lee JC. Mesh migration into the sigmoid colon mimics a colon tumor, a rare complication after herniorrhaphy: case report. Int J Color Dis. 2017;32(1):155-7.

14. Millas SG, Mesar T, Patel RJ. Chronic abdominal pain after ventral hernia due to mesh migration and erosion into the sigmoid colon from a distant site: a case report and review of literature. Hernia. 2015;19(5):849-52.

15. Yilmaz I, Karakaș DO, Sucullu I, Ozdemir Y, Yucel E. A rare cause of mechanical bowel obstruction: mesh migration. Hernia. 2013;17(2):267-9.

16. Schug-Pass C, Lippert H, Kockerling F. Mesh fixation with fibrin glue (Tissucol/Tisseel) in hernia repair dependent on the mesh structure-is there an optimum fibrin-mesh combination?-investigations on a biomechanical model. Langenbeck's Arch Surg. 2010;395(5):569-74.

17. Mayer F, Niebuhr H, Lechner M, Dinnewitzer A, Köhler G, Hukauf $\mathrm{M}$, et al. When is mesh fixation in TAPP-repair of primary inguinal hernia repair necessary? The register-based analysis of 11,230 cases. Surg Endosc. 2016;30(10):4363-71.

18. Donahue JG, et al. Gastric and duodenal safety of daily alendronate. JAMA Intern Med. 2002;162(8):936-42.

19. Agresta F, Bedin N. Transabdominal laparoscopic inguinal hernia repair: is there a place for biological mesh? Hernia. 2008;12(6): 609-12.

20. Kockerling F, et al. Biological meshes for inguinal hernia repair review of the literature. Front Surg. 2015;2:48.

Publisher's Note Springer Nature remains neutral with regard to jurisdictional claims in published maps and institutional affiliations. 